\title{
Sombreamento de cafeeiros durante o desenvolvimento das gemas florais e seus efeitos sobre a frutificação e produção
}

\author{
Shading of coffee plants during floral buds development and its effects on fructification and production
}

\author{
Heverly Morais $^{\mathrm{I}^{*}}$ Paulo Henrique Caramori $^{\mathrm{I}}$ Mirian Sei Koguishi ${ }^{\mathrm{II}}$ José Carlos Gomes $^{\mathrm{I}}$ \\ Ana Maria de Arruda Ribeiro ${ }^{\mathrm{II}}$
}

\section{RESUMO}

O objetivo neste trabalho foi avaliar a influência do sombreamento, durante o período de desenvolvimento de gemas florais, sobre o desenvolvimento e maturação dos frutos, produção e tamanho dos grãos de Coffea arabica $L$. O experimento foi conduzido com cafeeiros adultos no Município de Londrina, os quais foram sombreados nos meses de abril, maio, junho, julho e agosto de 2004, com malhas de sombrite com $50 \%$ de sombreamento. As coberturas foram retiradas, simultaneamente, no início de outubro do mesmo ano. A interceptação de $50 \%$ da radiação incidente sobre o dossel dos cafeeiros em diferentes épocas do desenvolvimento de gemas florais não alterou o crescimento e o desenvolvimento dos frutos. As altas temperaturas e a deficiência hídrica anteciparam a maturação dos frutos, porém sem diferenças significativas nos estádios de maturação entre os tratamentos. O sombreamento, em diferentes épocas, também não afetou a produção e o tamanho dos grãos.

Palavras-chave: Coffea arabica, sombreamento artificial, sombrite, produtividade, maturação.

\section{ABSTRACT}

The objective of this paper was to evaluate the influence of shading during the period of floral buds development on the growth and maturation of coffee fruits, production and grain size of Coffea arabica $L$. The experiment was carried out in Londrina, PR, Brazil. Plots of adult coffee plants were shaded monthly in April, May, June, July and August of 2004 using shading screens with 50\% of porosity. The coverings were removed simultaneously at the beginning of October of the same year. The interception of $50 \%$ of incident radiation over the coffee canopy in different periods of floral buds development did not alter fruit growth and development. Fruit maturation was anticipated due to high temperatures and water deficit, however without significant differences on maturation periods among treatments. Grain yield and size were not affected by shading in the different periods.

Key words: Coffea arabica, artificial shading, shading screens, productivity, maturation.

\section{INTRODUÇÃO}

O sombreamento de cafezais é uma técnica tradicionalmente utilizada em países da América Latina, como Colômbia, Costa Rica, Equador e Guatemala. Nesses países, o cultivo a pleno sol geralmente não é recomendado, pois apresenta maior risco econômico e menor sustentabilidade, com exceção dos locais que possuem condições ótimas para a produção e qualidade do café, como cultivos em altitudes acima de $1200 \mathrm{~m}$ com boa disponibilidade hídrica e nutricional (VAAST et al., 2005). No Brasil, apesar de predominar o cultivo a pleno sol, a técnica de sombreamento de cafezais tem se expandido em virtude da necessidade de se produzir com menor uso de insumos, como no sistema orgânico. Outra razão é a possibilidade de renda adicional para o agricultor e manutenção do sistema equilibrado, com preservação da biodiversidade, solo, qualidade dos recursos hídricos e seqüestro de carbono. Para o cafeeiro, o sombreamento protege contra temperaturas

IInstituto Agronômico do Paraná (IAPAR), Rod. Celso Garcia Cid, km 375, CP 481, 86001-970, Londrina, PR, Brasil. E-mail: heverly@iapar.br.*Autor para correspondência.

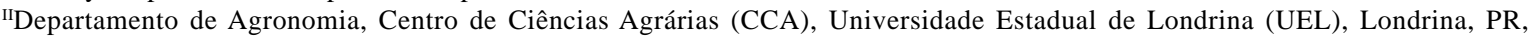
Brasil. 
extremas, reduz a bienalidade de produção, a incidência de seca dos ponteiros e melhora a qualidade do café (RENA \& MAESTRI, 1985; VAAST et al., 2005). Nas regiões brasileiras produtoras de café que estão sujeitas às geadas, o sombreamento tem a vantagem adicional de proteção contra baixas temperaturas, evitando perdas na produção e prejuízos para os cafeicultores.

Todavia, o sombreamento modifica o microclima no qual o cafeeiro se encontra e, dependendo da intensidade e duração, ocasiona mudanças fisiológicas, anatômicas e reprodutivas nas plantas, podendo afetar negativamente a produção (MORAIS, 2003). A produtividade de uma cultura, além de sua expressão genética e outras condições como status nutricional, suprimento hídrico, sanidade, controle de ervas daninhas e características do solo, também é resultado da eficiência do aproveitamento da radiação fotossintética. A esse respeito FERERES (1995) relata que a produção das culturas depende de três processos: interceptação da radiação solar incidente em um nível adequado pelos órgãos fotossintéticos da planta; conversão da energia nas ligações químicas dos produtos fotossintetizados e, por último, translocação destes nos distintos órgãos da planta. A eficiência fotossintética será maior na medida em que as condições ambientais (solo e clima) forem adequadas. Segundo MUSCHLER (1997), o sucesso do sombreamento depende, principalmente, das condições edáficas e da altitude. Em condições ambientais adequadas e com a utilização intensiva de insumos, plantios a pleno sol, usualmente, sobrepõemse aos arborizados em termos de produção (BEER et al., 1998). Em contrapartida, o sombreamento pode alterar o ciclo reprodutivo do cafeeiro e beneficiá-lo em termos de redução da bienalidade da produção, aumento da longevidade da planta e qualidade dos frutos. Vários estudos têm demonstrado que a altitude e o sombreamento incrementam a qualidade do café, à medida que as condições climáticas amenas prolongam a maturação dos frutos, produzindo grãos maiores e superiores acúmulos de açúcares e sólidos solúveis (VAAST, et al., 2005; LUNZ, 2006).

É importante destacar que, além do espaçamento entre árvores, outros aspectos referentes ao manejo da sombra, como época e duração, também devem ser considerados e avaliados, a fim de se conhecerem os limites do sombreamento, seleção de espécies arbustivas e/ou arbóreas e épocas de podas, de tal forma que favoreça a produção e ao mesmo tempo proporcione aos cafeeiros os outros benefícios do sombreamento, anteriormente citados.

O objetivo neste trabalho foi avaliar a influência do sombreamento artificial, durante o período de desenvolvimento de gemas reprodutivas (outono/ inverno), sobre o desenvolvimento e maturação dos frutos, produção e tamanho dos grãos de Coffea arabica.

\section{MATERIAL E MÉTODOS}

O experimento foi desenvolvido no campo experimental do IAPAR, em Londrina, PR, cujas coordenadas geográficas são: altitude $610 \mathrm{~m}$, latitude $23^{\circ} 23^{\prime}$ S e longitude $50^{\circ} 11^{\prime} \mathrm{W}$. O clima da região é do tipo Cfa, caracterizado como subtropical úmido com verão quente, segundo a classificação de Köppen. A temperatura média anual é de $21^{\circ} \mathrm{C}$, sendo a média do mês mais quente (janeiro) de $24^{\circ} \mathrm{C}$ e a média do mês mais frio (junho) $17^{\circ} \mathrm{C}$. A precipitação média anual é de $1.500 \mathrm{~mm}$, sendo dezembro, janeiro e fevereiro os meses mais chuvosos e junho, julho e agosto os mais secos (CAVIGLIONE et al., 2000).

Foram avaliados cafeeiros da espécie Coffea arabica da cultivar 'IAPAR 59', plantados em junho de 1993, em um espaçamento de $2,5 \mathrm{~m}$ entre linhas e $1,5 \mathrm{~m}$ entre plantas, com duas plantas por cova. Em outubro de 2000 foram recepados em decorrência de geada. Os tratamentos constituíram de cobertura das plantas no início de abril (T1), maio (T2), junho (T3), julho (T4) e agosto (T5) de 2004, utilizando malhas do tipo “sombrite" com $50 \%$ de sombreamento e dimensão de $12 \mathrm{~m}$ de comprimento, 7,5m de largura e 2,5m de altura, mantendo-se um tratamento sem cobertura. As coberturas foram retiradas, simultaneamente, no início de outubro do mesmo ano. O delineamento experimental utilizado foi inteiramente casualizado, com quatro repetições. Em cada parcela, foram cobertas três linhas de cafeeiros com oito covas cada. Foram marcados e avaliados em dois ramos, oito nós florais produtivos: quatro voltados para a direção norte e outros quatro para a direção sul, localizados no terço superior de quatro plantas da linha central. As avaliações foram feitas por meio de observações visuais externas do tamanho, quantidade e coloração dos frutos nas seguintes datas: 20/10/2004, 08/11/2004, 24/11/2004, 05/ 
12/2004, 26/01/2005, 15/03/2005, 29/03/2005, 12/04/2005, 26/04/2005, 10/05/2005 e 20/05/2005.

O crescimento dos frutos foi classificado como: $\mathrm{F}_{1}$ - frutos de até $3 \mathrm{~mm}$ de comprimento; $\mathrm{F}_{2}-3,1$ a 4mm; $F_{3}-4,1$ a 5mm; $F_{4}-5,1$ a $10 \mathrm{~mm} ; F_{5}-10$ a $15 \mathrm{~mm}$; $\mathrm{F}_{6}$ - maior que $15 \mathrm{~mm}$. Na fase de maturação fisiológica os frutos foram avaliados de acordo com a coloração: verde - frutos verdes; verde cana - frutos amarelados; cereja - frutos vermelhos ou iniciando a coloração vermelha; passa - frutos vermelho-escuro; e seco frutos desidratados com coloração externa escura. Para avaliação da produção foram colhidas doze plantas/ parcela, as quais foram pesadas imediatamente após a colheita para se obterem os pesos de café da "roça". Para determinação do peso de café em coco, foram retirados dois quilos de cada parcela e secados em uma estufa.

Após o beneficiamento foram retiradas amostras de duzentos gramas de grãos de cada parcela, que passaram por uma máquina elétrica vibratória contendo peneiras de malhas de diferentes diâmetros (18, 17, 16, 15, 14, 13mm), grãos moca e “fundo”. Os grãos tipo moca são de formato ovóide com ranhura central no sentido longitudinal (BRASIL, 2003). Os grãos classificados com "fundo" foram aqueles não retidos em nenhuma das peneiras e recolhidos em uma bandeja situada na parte basal da máquina. Em seguida pesaram-se, separadamente, os grãos contidos em cada extrato.

A maturação dos frutos foi avaliada por meio de estatística descritiva, determinando as freqüências de cada estádio de desenvolvimento em relação ao total de estádios em cada avaliação. Os dados de produção e peneiras foram submetidos à comparação de médias pelo teste estatístico de Tukey a 5\% de probabilidade de erro.

\section{RESULTADOS E DISCUSSÃO}

O número de frutos nos diferentes estádios de crescimento, avaliados no período de outubro de 2004 a janeiro de 2005, é apresentado na tabela 1 . A média dos tratamentos para o número total de frutos foi de 1248 em 20/10 e 1652 em 09/11, ou seja, houve um acréscimo médio de 404 frutos por tratamento. Esse aumento do número de frutos ocorreu devido ao segundo florescimento, no qual foram quantificadas

Tabela 1 - Número de frutos, em diferentes estádios de crescimento ( $F_{1}$ a $\left.F_{6}\right)$, de cafeeiros sombreados artificialmente em diferentes épocas.

\begin{tabular}{|c|c|c|c|c|c|c|c|}
\hline Data & Estádio & $\mathrm{T} 1$ & $\mathrm{~T} 2$ & T3 & $\mathrm{T} 4$ & $\mathrm{~T} 5$ & Sem cobertura \\
\hline $20 / 10 / 2004$ & $\mathrm{~F} 1$ & 1542 & 1004 & 915 & 1345 & 1232 & 1451 \\
\hline \multirow{3}{*}{ 09/11/2004 } & $\mathrm{F} 1$ & 438 & 728 & 401 & 499 & 399 & 580 \\
\hline & $\mathrm{F} 2$ & 1457 & 852 & 863 & 1284 & 1146 & 1266 \\
\hline & Total & 1895 & 1580 & 1264 & 1783 & 1545 & 1846 \\
\hline \multirow{5}{*}{$24 / 11 / 2004$} & $\mathrm{~F} 1$ & 179 & 176 & 88 & 72 & 50 & 252 \\
\hline & F2 & 303 & 536 & 319 & 420 & 313 & 289 \\
\hline & F3 & 1184 & 744 & 760 & 1139 & 973 & 1154 \\
\hline & F4 & 207 & 120 & 92 & 132 & 169 & 76 \\
\hline & Total & 1873 & 1576 & 1259 & 1763 & 1505 & 1771 \\
\hline \multirow{6}{*}{ 05/12/2004 } & $\mathrm{F} 1$ & 75 & 85 & 110 & 92 & 45 & 126 \\
\hline & $\mathrm{F} 2$ & 324 & 518 & 311 & 341 & 281 & 348 \\
\hline & F3 & 842 & 452 & 135 & 288 & 199 & 780 \\
\hline & $\mathrm{F} 4$ & 433 & 335 & 381 & 771 & 680 & 441 \\
\hline & F5 & 162 & 164 & 308 & 245 & 247 & 4 \\
\hline & Total & 1836 & 1554 & 1245 & 1737 & 1452 & 1699 \\
\hline \multirow{6}{*}{$26 / 01 / 2005$} & $\mathrm{~F} 2$ & 48 & 40 & 19 & 16 & 20 & 32 \\
\hline & F3 & 18 & 34 & 33 & 21 & 13 & 13 \\
\hline & F4 & 8 & 17 & 31 & 39 & 6 & 38 \\
\hline & F5 & 88 & 95 & 102 & 209 & 27 & 217 \\
\hline & F6 & 1602 & 1306 & 1037 & 1340 & 1285 & 1150 \\
\hline & Total & 1764 & 1492 & 1222 & 1625 & 1351 & 1450 \\
\hline
\end{tabular}

Ciência Rural, v.39, n.2, mar-abr, 2009. 
383 flores por tratamento, em média. O cafeeiro tem o crescimento vegetativo e reprodutivo simultâneo, à medida que os ramos crescem as gemas se diferenciam, ocorrendo mais de uma florada e, conseqüentemente, frutos em diferentes estádios no mesmo ramo e até no mesmo nó. Na avaliação do dia 24/11 o número de frutos estabilizou (média de 1625). Já nas avaliações seguintes, nos dias 05/12 e 26/01, a média dos tratamentos diminuiu para 1587 e 1484, respectivamente. A abscisão de frutos é um aspecto comum no cafeeiro, mas ainda não totalmente esclarecido. Neste trabalho não foram feitas avaliações endógenas hormonais, mas as épocas de queda dos frutos condizem com os resultados de OPILLE (1979), que analisou o conteúdo endógeno de fitormônios ao longo do desenvolvimento dos frutos de cafeeiros no Quênia e observou que nos estádios de desenvolvimento dos frutos correspondentes a $\mathrm{F}_{3}$ e $\mathrm{F}_{4}$, a concentração de giberelinas foi baixa e do ácido abscísico alta. O autor supôs que a combinação de fitormônios poderia ser responsável pela queda de frutos mesmo em condições favoráveis de umidade.

Na primeira avaliação (20/10) todos os frutos estavam no estádio $\mathrm{F}_{1}$. No dia 09/11 havia frutos nos estádios $\mathrm{F}_{1}$ e $\mathrm{F}_{2}$, ou seja, seis semanas após o primeiro florescimento todos os frutos não tinham diâmetros maiores que 4mm. Esses resultados condizem com CANNELL (1971), o qual observou que, durante as 5-6 semanas posteriores à antese, os frutos exibem um crescimento lento. Isso ocorreu porque os frutos são incapazes de competir por nutrientes com o crescimento vegetativo, que é rico em giberelinas (KUMAR, 1979).

No dia 24/11, apenas duas semanas após a avaliação anterior, já havia frutos nos estádios $\mathrm{F}_{3}$ e $\mathrm{F}_{4}$. Esse resultado corrobora com SALAZAR et al. (1994), que observaram que, a partir desse estádio, os frutos se expandem rapidamente até atingirem seu tamanho máximo, em torno da $16^{\mathrm{a}}$ semana $\left(\mathrm{F}_{6}\right)$.

Na avaliação do dia 05/12 não foi observada tendência de concentração de um estádio de crescimento entre os tratamentos. Isso ocorreu devido aos vários florescimentos associados à queda dos frutos e competição por nutrientes entre eles, fazendo com que frutos da mesma planta, mesmo ramo e até mesmo nó tivessem tamanhos diferentes. Na avaliação do mês de janeiro, a maioria dos frutos já estava na fase final de enchimento $\left(\mathrm{F}_{6}\right)$, em todos os tratamentos.
Não houve diferença estatística significativa entre os tratamentos para o número total de frutos. Também não foi evidenciada nenhuma tendência de concentração de determinado estádio de crescimento do fruto entre os tratamentos. Isso ocorreu porque durante essa fase todas as plantas estavam na condição de pleno sol, uma vez que as coberturas foram retiradas logo após o florescimento e a interferência do sombreamento nos frutos se dá por sombreamento direto sobre os cafeeiros no período de frutificação.

Houve maturação antecipada dos frutos dos cafeeiros (Tabela 2). A cultivar 'IAPAR 59' é classificada como precoce e a maturação e a colheita ocorrem normalmente a partir do final de junho, mas neste experimento ocorreu no final de maio. Isso aconteceu devido às altas temperaturas e deficiência hídrica registrada no final da maturação (Figura 1). Segundo KUMAR (1979), o tempo necessário para a completa maturação dos frutos varia de acordo com as condições climáticas vigentes e o genótipo da cultivar.

Na evolução da maturação dos frutos notase uniformidade entre os tratamentos em todas as avaliações. Isso indica ausência de influência do sombreamento artificial sobre a maturação dos frutos nos diferentes períodos durante o desenvolvimento da gema floral. Vários autores observaram que a maturação ocorre mais lentamente em frutos de cafeeiros sombreados, comparado com cafeeiros cultivados a pleno sol (MORAIS, 2003; VAAST et al., 2005; LUNZ, 2006). Todavia, em todos esses trabalhos, o sombreamento teve influência direta no crescimento e maturação dos frutos, ou seja, o estádio de frutificação ocorreu sob sombra, diferentemente deste experimento em que as coberturas foram retiradas após a floração.

Na tabela 3 são apresentados os valores médios de produção de 2005, referentes à colheita de doze plantas por parcela. Verifica-se que as produções médias de café da roça, coco e beneficiado não diferiram, estatisticamente, entre os tratamentos. Isso significa que o sombreamento nas diferentes épocas, durante o desenvolvimento das gemas florais, não interferiu na produção do ano seguinte (2005). Analisando o ciclo fenológico do cafeeiro, constata-se que o crescimento dos nós produtivos ocorreu na estação anterior ao sombreamento, portanto a pleno sol e, de acordo com GOUVEIA (1984), a produção depende da interação entre o número de nós formados na estação de crescimento e as condições ambientais favoráveis à

Ciência Rural, v.39, n.2, mar-abr, 2009. 
Tabela 2 - Maturação de frutos de cafeeiros sombreados artificialmente em diferentes épocas.

\begin{tabular}{|c|c|c|c|c|c|c|c|}
\hline Data & Estádio & $\mathrm{T} 1(\%)$ & $\mathrm{T} 2(\%)$ & ТЗ(\%) & $\mathrm{T} 4(\%)$ & T5(\%) & Sem cobertura (\%) \\
\hline \multirow{5}{*}{$15 / 03$} & Verde & 72 & 90 & 90 & 88 & 87 & 81 \\
\hline & Cana & 24 & 6 & 6 & 7 & 9 & 13 \\
\hline & Cereja & 3 & 2 & 4 & 3 & 3 & 5 \\
\hline & Passa & 0 & 0 & 0 & 0 & 0 & 0 \\
\hline & Seco & 1 & 1 & 1 & 2 & 1 & 1 \\
\hline \multirow{5}{*}{$29 / 03$} & Verde & 53 & 81 & 77 & 77 & 73 & 67 \\
\hline & Cana & 31 & 12 & 10 & 11 & 15 & 17 \\
\hline & Cereja & 10 & 2 & 9 & 7 & 8 & 6 \\
\hline & Passa & 0 & 0 & 1 & 0 & 0 & 0 \\
\hline & Seco & 5 & 5 & 2 & 5 & 4 & 10 \\
\hline \multirow{5}{*}{$12 / 04$} & Verde & 32 & 65 & 43 & 46 & 47 & 40 \\
\hline & Cana & 33 & 16 & 23 & 20 & 17 & 28 \\
\hline & Cereja & 26 & 11 & 27 & 23 & 29 & 14 \\
\hline & Passa & 1 & 0 & 2 & 1 & 1 & 0 \\
\hline & Seco & 8 & 8 & 5 & 10 & 7 & 18 \\
\hline \multirow{5}{*}{$20 / 05$} & Verde & 14 & 41 & 28 & 27 & 25 & 18 \\
\hline & Cana & 24 & 28 & 11 & 16 & 15 & 28 \\
\hline & Cereja & 53 & 28 & 52 & 50 & 53 & 44 \\
\hline & Passa & 4 & 0 & 2 & 0 & 1 & 0 \\
\hline & Seco & 5 & 2 & 7 & 7 & 6 & 10 \\
\hline
\end{tabular}

diferenciação das gemas florais. Em estudos com sombreamento artificial de cafeeiros durante todo o ciclo reprodutivo, JARAMILLO et al. (2003) encontraram maior produção em plantas sob $48 \%$ de bloqueio da radiação fotossinteticamente ativa com menor quantidade de fertilizantes. Em contrapartida,

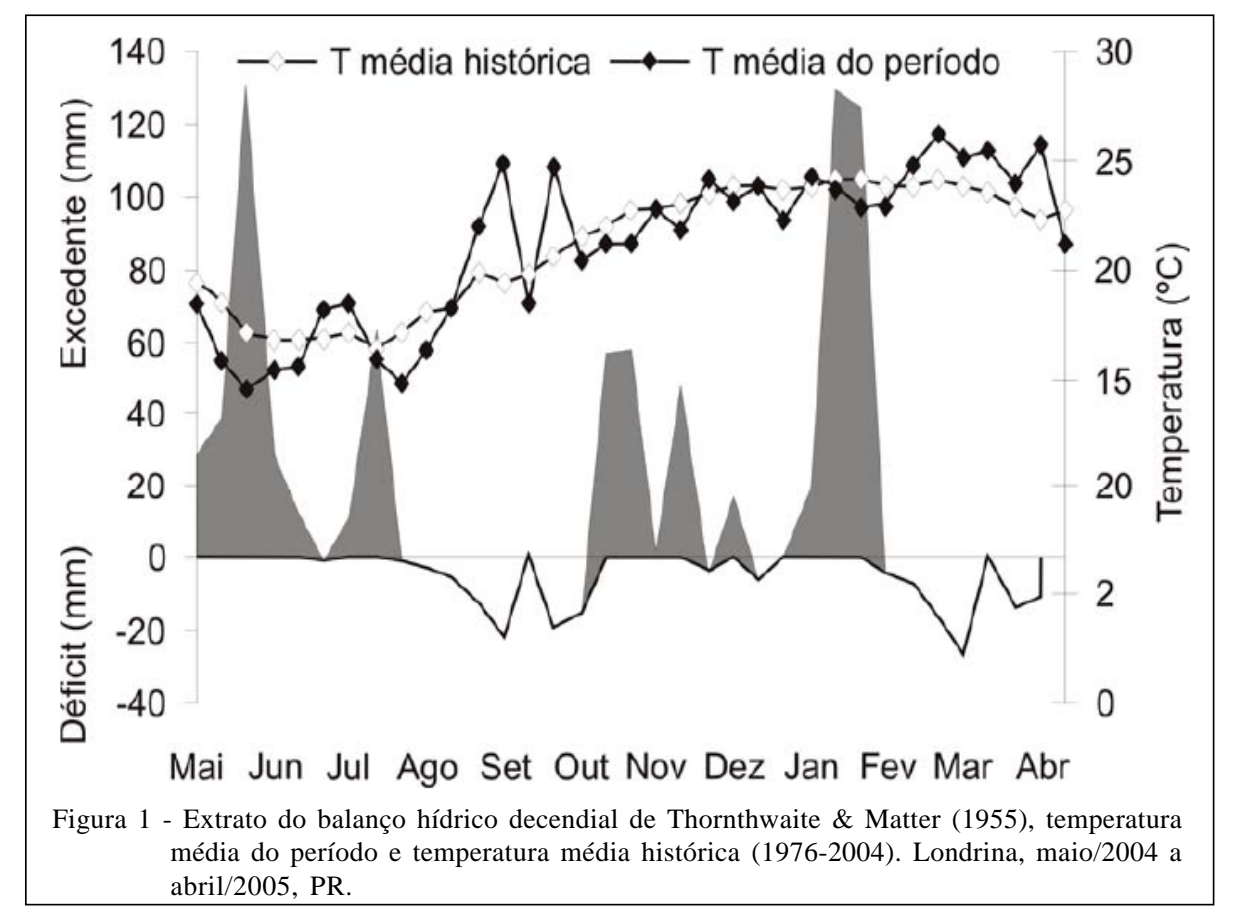

Ciência Rural, v.39, n.2, mar-abr, 2009. 
Tabela 3 - Produção ( $\mathrm{Kg}^{-1}$.parcela) de cafeeiros sombreados artificialmente em abril (T1), maio (T2), junho (T3), julho (T4), agosto (T5), safra 2005.

\begin{tabular}{lccc}
\hline Tratamento & Café da roça & Café em coco & Café beneficiado \\
\hline T1 & $50,39 \mathrm{a}^{*}$ & $26,45 \mathrm{a}$ & $12,37 \mathrm{a}$ \\
T2 & $53,51 \mathrm{a}$ & $21,73 \mathrm{a}$ & $12,45 \mathrm{a}$ \\
T3 & $53,94 \mathrm{a}$ & $24,85 \mathrm{a}$ & $13,84 \mathrm{a}$ \\
T4 & $56,07 \mathrm{a}$ & $26,27 \mathrm{a}$ & $14,63 \mathrm{a}$ \\
T5 & $54,19 \mathrm{a}$ & $26,91 \mathrm{a}$ & $14,64 \mathrm{a}$ \\
Sem cobertura & $52,19 \mathrm{a}$ & $25,20 \mathrm{a}$ & $13,93 \mathrm{a}$ \\
CV\% & 13,07 & 15,20 & 13,97 \\
DMS & 16,03 & 8,81 & 4,38 \\
\hline
\end{tabular}

*Médias com a mesma letra na coluna não diferem entre si, pelo teste de Tukey, a 5\% de probabilidade de erro.

CARELLI et al. (2001) observaram redução na produção acumulada de dois anos em condições moderadas de sombreamento (50\% e 30\%). Em condições de sombreamento natural, LUNZ (2006) encontrou maiores produtividades de café consorciados com seringueiras em Piracicaba, SP, até um limite de 70\% de irradiância. No México, SOTO-PINTO et al. (2000) não observaram redução na produtividade de café por planta até um limite de 50\% de sombreamento. Sistemas de cultivos arborizados e consorciados com atenuações de, aproximadamente, $20 \%$ de radiação solar global não produziram efeitos negativos na produção de café (PEETERS et al., 2002; PEZZOPANE, 2004). Por outro lado, MORAIS et al. (2006) comprovaram que o sombreamento excessivo em períodos prolongados, reduz significativamente a produção.

Em todos os tratamentos (Tabela 4) a maior parte dos grãos de café ficou retida na peneira média (grãos com 15 a 16mm de diâmetros), característica própria da cultivar avaliada. Isso mostra que não houve efeito dos tratamentos para tamanho de grão do café. Resultados encontrados por MANOEL et al. (2002), VAAST et al. (2005) e LUNZ (2006) mostraram grãos maiores em cafeeiros sombreados durante todo o ciclo reprodutivo em relação àqueles cultivados a pleno sol. Isso indica que o sombreamento, durante o período de formação dos frutos, tem influência direta sobre tamanho dos grãos.

\section{CONCLUSÃO}

A interceptação de $50 \%$ da radiação incidente sobre cafeeiros, em diferentes épocas do desenvolvimento de gemas florais, não alterou o crescimento, a maturação, a produção e o tamanho dos grãos.

Tabela 4 - Massa ( $\mathrm{g}^{-1}$.parcela) e porcentagem (\%) de grãos beneficiados de cafeeiros sombreados artificialmente em diferentes épocas.

\begin{tabular}{|c|c|c|c|c|c|c|c|c|}
\hline & $\mathrm{T} 1$ & $\mathrm{~T} 2$ & T3 & $\mathrm{T} 4$ & T5 & $\begin{array}{c}\text { Sem } \\
\text { cobertura }\end{array}$ & $\mathrm{CV} \%$ & DMS \\
\hline Peneira grande (18 e 17) & $\begin{array}{l}12,92 a^{*} \\
(6 \%)\end{array}$ & $\begin{array}{l}12,15 \text { a } \\
(6 \%)\end{array}$ & $\begin{array}{l}12,92 \text { a } \\
(6 \%)\end{array}$ & $\begin{array}{l}8,15 \text { a } \\
(4 \%)\end{array}$ & $\begin{array}{l}14,37 \text { a } \\
(7 \%)\end{array}$ & $\begin{array}{l}11,12 \text { a } \\
(6 \%)\end{array}$ & 40,04 & 10,98 \\
\hline Peneira média (16 e 15) & $\begin{array}{l}106,15 \text { a } \\
(53 \%)\end{array}$ & $\begin{array}{l}98,78 \text { a } \\
(49 \%)\end{array}$ & $\begin{array}{l}109,65 \text { a } \\
(55 \%)\end{array}$ & $\begin{array}{l}96,44 \mathrm{a} \\
(48 \%)\end{array}$ & $\begin{array}{l}102,29 \text { a } \\
(50 \%)\end{array}$ & $\begin{array}{l}85,89 \text { a } \\
(43 \%)\end{array}$ & 12,18 & 27,96 \\
\hline Peneira pequena (14 e13) & $\begin{array}{l}41,69 \text { a } \\
(21 \%)\end{array}$ & $\begin{array}{l}47,57 \text { a } \\
(23 \%)\end{array}$ & $\begin{array}{l}39,32 \mathrm{a} \\
(20 \%)\end{array}$ & $\begin{array}{l}45,35 \text { a } \\
(23 \%)\end{array}$ & $\begin{array}{l}39,95 \text { a } \\
(20 \%)\end{array}$ & $\begin{array}{l}48,92 \mathrm{a} \\
(25 \%)\end{array}$ & 17,23 & 17,34 \\
\hline Grãos moca & $\begin{array}{l}18,91 \text { a } \\
(9 \%)\end{array}$ & $\begin{array}{l}17,07 \text { a } \\
(8 \%)\end{array}$ & $\begin{array}{l}18,83 \text { a } \\
(9 \%)\end{array}$ & $\begin{array}{l}23,15 \text { a } \\
(12 \%)\end{array}$ & $\begin{array}{l}25,05 \text { a } \\
(12 \%)\end{array}$ & $\begin{array}{l}18,21 \text { a } \\
(9 \%)\end{array}$ & 41,31 & 19,18 \\
\hline Fundo & $\begin{array}{l}20,91 \text { a } \\
(10 \%)\end{array}$ & $\begin{array}{l}28,02 \text { a } \\
(14 \%)\end{array}$ & $\begin{array}{l}18,06 \text { b } \\
(9 \%)\end{array}$ & $\begin{array}{l}26,13 \text { a } \\
(13 \%)\end{array}$ & $\begin{array}{l}20,96 \text { a } \\
(10 \%)\end{array}$ & $\begin{array}{l}33,54 \text { a } \\
(17 \%)\end{array}$ & 26,03 & 14,71 \\
\hline
\end{tabular}

*Médias com a mesma letra na linha não diferem entre si, pelo teste de Tukey, a 5\% de probabilidade de erro.

Ciência Rural, v.39, n.2, mar-abr, 2009. 


\section{REFERÊNCIAS}

BEER, J. et al. Shade management in coffee and cacao plantations. Agroforestry Systems, v.38, p.39-164, 1998.

BRASIL. Decreto-Lei nº̣. 4.629, de 21 de março de 2003. Estabelece critérios técnicos de identidade e de qualidade para a classificação do café beneficiado grão cru. Diário Oficial da República Federativa do Brasil de 13-06-2003, Brasília, p.4. Seção 1. Online. Disponível na internet http:// www.agricultura.gov.br/legislação/SISLEGIS .

CANNELL, M.G.R. Effect of the presence of fruits on net photosynthesis. Annual Report Coffee Research Station, v.6, p.41-42, 1971.

CARELLI, M.L.C. et al. Efeitos de níveis de sombreamento no crescimento e produtividade do cafeeiro. In: SIMPÓSIO DE PESQUISA DOS CAFÉS DO BRASIL, 2., 2001, Vitória, ES. Resumos... Vitória: EMBRAPA, 2001. p.16.

CAViglione, J.H. et al. Cartas climáticas do Paraná. Londrina: IAPAR, 2000. CD.

FERERES, C.E. Productividad de los sistemas de cultivo en invernadero. In: SIMPOSIUM IBEROAMERICANO SOBRE APLICACIÓN DE LOS PLÁSTICOS EM LAS TECNOLOGIAS AGRÁRIAS, 1995, Almeria. Actas... Almeria: FIAPA/CEPLA, 1995. p.287-296.

JARAMILLO, B.C. et al. Desenvolvimento reprodutivo e produção de cafeeiros sob níveis de sombreamento e adubação. In. SIMPÓSIO DE PESQUISA DOS CAFÉS DO BRASIL, 3., 2003, Porto Seguro, BA. Resumos... Porto Seguro: EMBRAPA, 2003. p.285-286.

KUMAR, D. Some aspects of plant-water-nutrient relationship in Coffea arabica L. Kenya Coffee, v.43, n.10, p.9-47, 1979.

LUNZ, A.M.P. Crescimento e produtividade do cafeeiro sombreado e a pleno sol. 2006. 94f. Tese (Doutorado em Agronomia) - Escola Superior de Agricultura Luiz de Queiroz, Piracicaba, SP.

GOUVEIA, N. Estudo da diferenciação e crescimento de gemas florais de Coffea arabica L.; observações sobre antese e maturação dos frutos. 1984. 237f. Dissertação (Mestrado em Ciências) - Universidade Estadual de Campinas, Campinas, SP.

MANOEL, R.M. et al. Influência da sombra de bananeira (Musa spp) na qualidade do café arábica (Coffea arabica L) cultivados sob sistema orgânico de produção. In: CONGRESSO BRASILEIRO DE PESQUISAS CAFEEIRAS, 28., 2002,
Caxambu. Trabalhos apresentados... Caxambu: MAA/ Procafé, 2002. p.299-300.

MORAIS, H. Efeito do sombreamento de cafeeiros (Coffea arabica L.) com guandu (Cajanus cajan (L) Millsp.) no Norte do Paraná. 2003. 118f. Dissertação (Mestrado em Agronomia) - Universidade Estadual de Londrina, Londrina, PR.

MORAIS, H. et al. Microclimatic characterization and productivity of coffee plants grown under shade of pigeonpea in Southern Brazil. Pesquisa Agropecuária Brasileira, v.41, n.5, p.763-770, 2006.

MUSCHLER, R.G. Sombra o sol para un cafetal sostenible: un nuevo enfoque de una vieja discusion. In: SIMPÓSIO LATINOAMERICANO DE CAFICULTURA, 18., 1997, San José Costa Rica. Anais... San José: Echeverri J and Zamora L (eds), 1997. p.471-476.

OPILLE, W.R. Hormonal relations in fruit growth and development of Coffea arabica L. Kenya Coffee, v.44, n.520, p.13-21, 1979.

PEETERS, L.Y.K. et al. Coffee production, timber and firewood in traditional and Ingá-shaded plantations in Southern México. Agriculture, Ecosystems and Environment, v.82, p.1-13, 2002.

PEZZOPANE, J.R.M. Avaliações microclimáticas, fenológicas e agronômicas em café arábica cultivado a pleno sol e consorciado com banana 'Prata Anã'. 2004. 136f. Tese (Doutorado em Agronomia) - Escola Superior de Agricultura Luiz de Queiroz, Piracicaba, SP.

RENA, A.B.; MAESTRI, M. Fisiologia do cafeeiro. Informe agropecuário, v.11, p.26-40, 1985.

SALAZAR, G.M.R. et al. Crecimiento del fruto de Coffea arabica var. Colômbia. Cenicafé, v.45, n.2, p.41-50, 1994.

SOTO-PINTO, L. et al. Shade effect on coffee production at the northern Tzeltal zone of the state of Chiapas, México. Agriculture, Ecosystems and Environment, v.80, p.6169, 2000.

VAAST, P. et al. Fruit thinning and shade improve bean characteristics and beverage quality of coffee (Coffea arabica L.) under optimal conditions. Journal of the Science of Food and Agriculture, v.86, n.2, p.197-204, 2005.

THORNTHWAITE, C.W.; MATHER, J.R. The water balance. Publication in Climatology, v.8, n.l, p.1-104, 1955. 